\title{
The decline rate of the production cross-section of superheavy nuclei with $Z=114-117$ at high excitation energies
}

\author{
J. Hong, \\ Department of Physics and Institute of Physics and Applied Physics, \\ Yonsei University, Seoul 03722, Korea \\ G. G. Adamian, \\ Joint Institute for Nuclear Research, Dubna 141980, Russia \\ N. V. Antonenko, \\ Joint Institute for Nuclear Research, Dubna 141980, Russia \\ Tomsk Polytechnic University, 634050 Tomsk, Russia \\ P. Jachimowicz, \\ Institute of Physics, University of Zielona Góra, \\ Szafrana 4a, 65516 Zielona Góra, Poland \\ M. Kowal \\ National Centre for Nuclear Research, \\ Pasteura 7, 02-093 Warsaw, Poland
}

(Dated: March 9, 2021) 


\begin{abstract}
The production cross sections of superheavy nuclei with charge numbers $114-117$ are predicted in the $(5-9) n$-evaporation channels of the ${ }^{48} \mathrm{Ca}$-induced complete fusion reactions for future experiments. The estimates of synthesis capabilities are based on a uniform and consistent set of input nuclear data provided by the multidimensional macroscopic-microscopic approach. The contributions of various factors to the final production cross section are discussed. As shown, the specific interplay between survival and fusion probabilities unexpectedly leads to a relatively slow decline of the total cross-sections with increasing excitation energy. This effect is supported by a favorable arrangement of fission barriers protecting the compound nucleus against splitting concerning energetic thresholds for the emission of successive neutrons. In particular, the probabilities of the formation of superheavy nuclei in the $5 n-, 6 n$-, and in some cases even in $7 n$-evaporation channels are still promising. This may offer a new opportunity for the future synthesis of unknown neutron-deficient superheavy isotopes.
\end{abstract}

PACS numbers: 25.70.Hi, 24.10.-i, 24.60.-k

Key words: Superheavy nuclei; Complete fusion reactions; Production of superheavy nuclei; $x n$-evaporation channels 
The question of what is the largest possible atomic number of a chemical element in the Periodic Table is still unsettled [1-4]. Currently, other unsolved problems are how to extend the chart of nuclides and how to explain the abundance distribution of elements in the Universe and in the Solar System [5]. The complete fusion reactions with ${ }^{48} \mathrm{Ca}$ beams and actinide targets have been successfully used to synthesize superheavy nuclei (SHN) Cn, Nh, Fl, Mc, Lv, Ts, Og with charge numbers $Z=112-118$ in the neutron evaporation channels ( $x n$-evaporation channels, where $x$ is the number of neutrons emitted) $[1,2$, $6-10]$ and allowed for slight approach to the so-called "the island of stability" of SHN [1-3]. Most of these SHN has been obtained in the $3 n$ - and $4 n$-evaporation channels. Only in the reactions ${ }^{48} \mathrm{Ca}+{ }^{242} \mathrm{Pu},{ }^{243} \mathrm{Am},{ }^{245} \mathrm{Cm}$ the evaporation residues have been detected in the $2 n$-evaporation channel. The nuclei ${ }^{285,287} \mathrm{Fl}$ have been also produced in the $5 n$-evaporation channel. During the transition from the $4 n$-evaporation channel to the $5 n$-evaporation channel, the crosssection dropped from about $(4-10)$ pb to about $(0.6-1)$ pb [2]. However, the question of how rapidly the evaporation residue cross section decreases with increasing beam energy is still open. In the present paper, we want to answer this question, bearing in mind the possibilities of already expanded/improved devices and soon possible experiments. Note that in our previous letter [11], the production cross sections of the SHN with charge numbers $Z=112-118$ were quite well described in $x n$-evaporation channels $(x=2-5)$ using the predictions of SHN properties from Refs. [12, 13]. The present article is then a natural continuation of these studies. Employing the same mass table of Refs. [12, 13] based on the microscopic-macroscopic (MM) method, we are going to predict the chances of producing new SHN in the $(5-9) n$-evaporation channels of the ${ }^{48} \mathrm{Ca}$-induced complete fusion reactions allowing much higher excitation energies. Such estimates and analyzes of the corresponding excitation functions, to the best of our knowledge, are unknown in the literature.

The evaporation residue cross section is factorized into three independent ingredients [11, $14-16]$ :

$$
\sigma_{s}\left(E_{\text {c.m. }}\right)=\sum_{J} \sigma_{c a p}\left(E_{\text {c.m. }}, J\right) P_{C N}\left(E_{\text {c.m. }}, J\right) W_{s}\left(E_{\text {c.m. }}, J\right) .
$$

In the evaporation channel, $s$ depends on the partial capture cross section $\sigma_{c a p}$ for the transition of the colliding nuclei over the entrance (Coulomb) barrier, the probability of CN formation $P_{C N}$ after capture and the survival probability $W_{s}$ of excited CN which estimates the competition between fission, neutron, and charged particles evaporation in the excited 
$\mathrm{CN}$. The formation of $\mathrm{CN}$ is calculated within the dinuclear system model in version as described in Refs. [11, 16]. This is well tested model with strong predictive power. In Eq. (1) the contributing angular momentum range is limited by $W_{s}$ and $P_{C N}$. In the case of highly fissile SHN, $W_{s}$ is a rather narrow function function of $J$ different from zero in the vicinity of $J=0$ for all bombarding energies $E_{\text {c.m. }}$.

Nuclear properties required for the correct estimation of the survival probability $\left(W_{s}\right)$ were systematically calculated within the multidimensional MM approach [12]. State-ofthe-art methods were used: minimization over many deformation parameters for minima and the imaginary water flow on many-deformation energy grids for saddles, including nonaxial and reflection-asymmetric shapes. Our systematic calculations include odd- $A$ and odd-odd nuclei with inner and outer fission barriers what is quite scarce in the literature. For nuclei with odd numbers of protons, neutrons, or both, we use a standard BCS method with blocking. One should emphasize that the MM method used here offers very good agreement with various existing experimental data (nuclear masses, decay energies, fission barriers, etc.) in a wide range of heaviest nuclei [12]. Decisive for the survival of newly

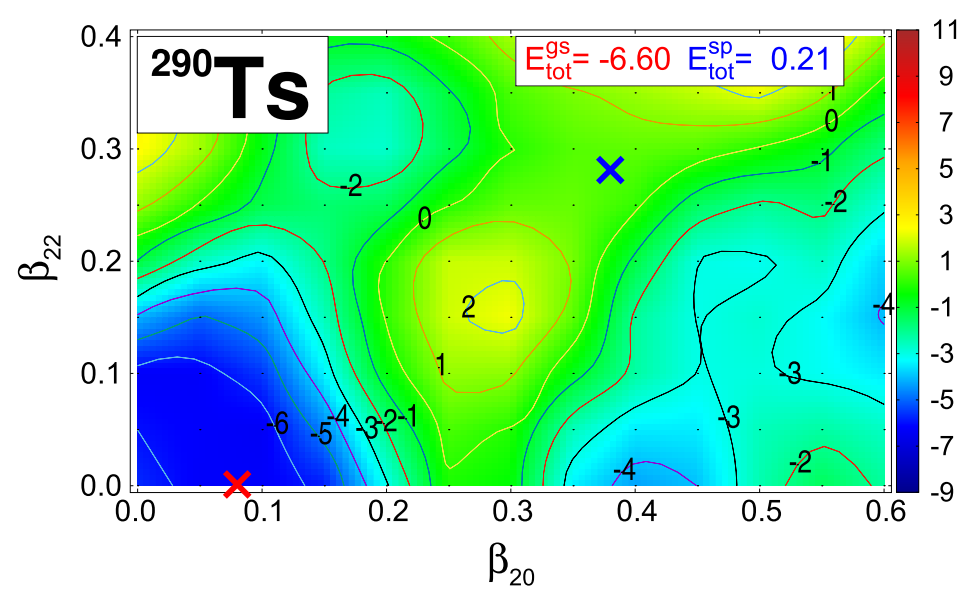

FIG. 1: The Potential Energy Surface (PES), $E-E_{m a c}\left(\right.$ sphere), for ${ }_{117}^{290} \mathrm{Ts}$. The crosses indicate the saddle point (blue) and the ground state (red).

created compound nucleus is a competition between fission and neutron emission process in the successive steps of the decay cascade. To put it simply, the resolution of this competition is determined by the energy thresholds - the fission barrier $B_{f}$ and the neutron separation energy $B_{n}$. An example of the potential energy surface allow to find these key values is shown in the Fig. [1 for the odd-odd ${ }_{117}^{290}$ Ts nucleus while energetic relation for decay thresholds is shown in Fig. 2, A value of $B_{f} / B_{n}$ greater than one just means that the nucleus is more 
protected against fission compared to the neutron emission. This is explicitly shown in Table

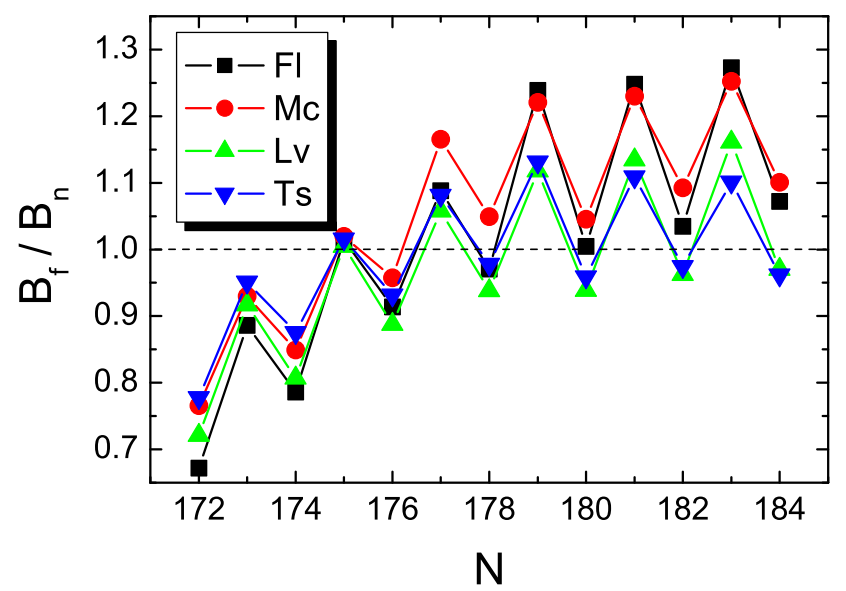

FIG. 2: The ratio $B_{f} / B_{n}$ vs the neutron number $N=A-Z$ for the $\mathrm{SHN}{ }_{114} \mathrm{Fl},{ }_{115} \mathrm{Mc},{ }_{116} \mathrm{Lv}$, ${ }_{117}$ Ts.

[1 where the difference $B_{f}-B_{n}$ - decisive for the survival probability of the newly formed SHN was calculated based on the new tables of masses and fission barriers [12]. Values greater than zero give (in the first order) an optimistic prediction of the new synthesis. The calculated excitation functions for $(5-9) n$-evaporation channels are presented in Figs. 3 and 4 for the complete fusion reactions ${ }^{48} \mathrm{Ca}+{ }^{242,244} \mathrm{Pu},{ }^{243} \mathrm{Am},{ }^{248} \mathrm{Cm},{ }^{249} \mathrm{Bk}$. As one can see, the production cross sections for the $(2-5) n$-evaporation channel are in quite a good agreement with the available experimental data. As mainly seen, the rather weak drop of the cross section with increasing excitation energy $E_{C N}^{*}$ is due to the interplay between the fusion $P_{C N}$ and the survival $W_{x n}$ probabilities, and due to a weak change of the difference between the fission barrier height and neutron separation energy at (5-9) steps of neutron evaporation as shown in Fig. 2 and in Table丩. Indeed, because the attenuation of the shell correction at high excitation energies, the fission barrier heights become close in $x n$ - and $(x+1) n$-evaporation channels. The compliance of the excitation functions obtained under the current approach for other target-projectile combinations leading to the already known SHN can be checked in Ref. [11]. Predictions for the production cross sections in high neutron emission channels are shown in the Fig. 4 for Mc, Lv, and Ts. One can see that according to our estimations, the production of unknown isotopes ${ }^{291} \mathrm{Ts}$ and ${ }^{290} \mathrm{Ts}$ in the $6 n$ - and $7 n$-evaporation channels, respectively, is still quite probable as the maxima of the cross sections are of the order 
TABLE I: Calculated $B_{f}-B_{n}$ based on Ref. [12].

\begin{tabular}{|c|c|c|c|c|}
\hline $\mathrm{N}$ & $\mathrm{Fl}$ & $\mathrm{Mc}$ & $\mathrm{Lv}$ & $\mathrm{Ts}$ \\
\hline 172 & -2.36 & -1.70 & -2.11 & -1.70 \\
173 & -0.74 & -0.47 & -0.56 & -0.35 \\
174 & -1.50 & -1.07 & -1.42 & -0.92 \\
175 & 0.09 & 0.13 & 0.03 & 0.11 \\
176 & -0.57 & -0.29 & -0.80 & -0.48 \\
177 & 0.54 & 1.06 & 0.37 & 0.55 \\
178 & -0.20 & 0.32 & -0.42 & -0.15 \\
179 & 1.30 & 1.28 & 0.70 & 0.80 \\
180 & 0.03 & 0.29 & -0.41 & -0.27 \\
181 & 1.32 & 1.31 & 0.77 & 0.66 \\
182 & 0.21 & 0.55 & -0.24 & -0.16 \\
183 & 1.41 & 1.36 & 0.88 & 0.58 \\
184 & 0.41 & 0.57 & -0.18 & -0.23 \\
\hline
\end{tabular}

of tenths of a picobarns $-\sigma_{6 n}^{\max } \approx 0.2 \mathrm{pb}$ and $\sigma_{7 n}^{\max } \approx 0.1 \mathrm{pb}$. Similarly optimistic are channels: $7 n$ in the reactions ${ }^{48} \mathrm{Ca}+{ }^{248} \mathrm{Cm}$ to produce the unknown isotope ${ }^{289} \mathrm{Lv}$ or $(5-7) n$ in reaction ${ }^{48} \mathrm{Ca}+{ }^{243} \mathrm{Am}$ which may still quite likely leads to new isotopes ${ }^{286} \mathrm{Mc}\left(\sigma_{5 n}^{\max } \approx 1\right.$ $\mathrm{pb}),{ }^{285} \mathrm{Mc}\left(\sigma_{6 n}^{\max } \approx 0.2 \mathrm{pb}\right)$ or to ${ }^{284} \mathrm{Mc}\left(\sigma_{7 n}^{\max } \approx 0.1 \mathrm{pb}\right)$. Note that for the production of unknown neutron-deficient isotope ${ }^{283} \mathrm{Fl}$, the hot fusion reaction ${ }^{48} \mathrm{Ca}+{ }^{242} \mathrm{Pu} \rightarrow{ }^{283} \mathrm{Fl}+7 n$ $\left(\sigma_{7 n}^{\max } \approx 0.1 \mathrm{pb}\right)$ looks superior to the cold fusion reaction ${ }^{76} \mathrm{Ge}+{ }^{208} \mathrm{~Pb} \rightarrow{ }^{283} \mathrm{Fl}+1 n$ for which $\sigma_{1 n}^{\max } \lesssim \sigma_{1 n}^{\exp }\left({ }^{70} \mathrm{Zn}+{ }^{209} \mathrm{Bi}\right) \approx 0.02 \mathrm{pb}[4]$.

As an example, Fig. 5 shows $P_{C N}$ and $W_{x n}$ for the ${ }^{48} \mathrm{Ca}+{ }^{244} \mathrm{Pu}$ reaction. With increasing excitation energy from $55 \mathrm{MeV}$ to $102 \mathrm{MeV}$, the value of $P_{C N}$ increases by about 6 times, while the value of $W_{x n}$ drops down by about 640 times and as a result, the production cross section decreased by more than 2 orders of magnitude. In the case of the ${ }^{48} \mathrm{Ca}+{ }^{249} \mathrm{Bk}$ reaction, the fusion probability grows by about 13 times, while the survival probability decreases by about $10^{4}$. It should be also noted that the capture cross section changes slightly in this case due to the limitation of the upper limit of angular momentum and that we observe a similar pattern for the other reactions in Figs. 3 and 4. A new fascinating 

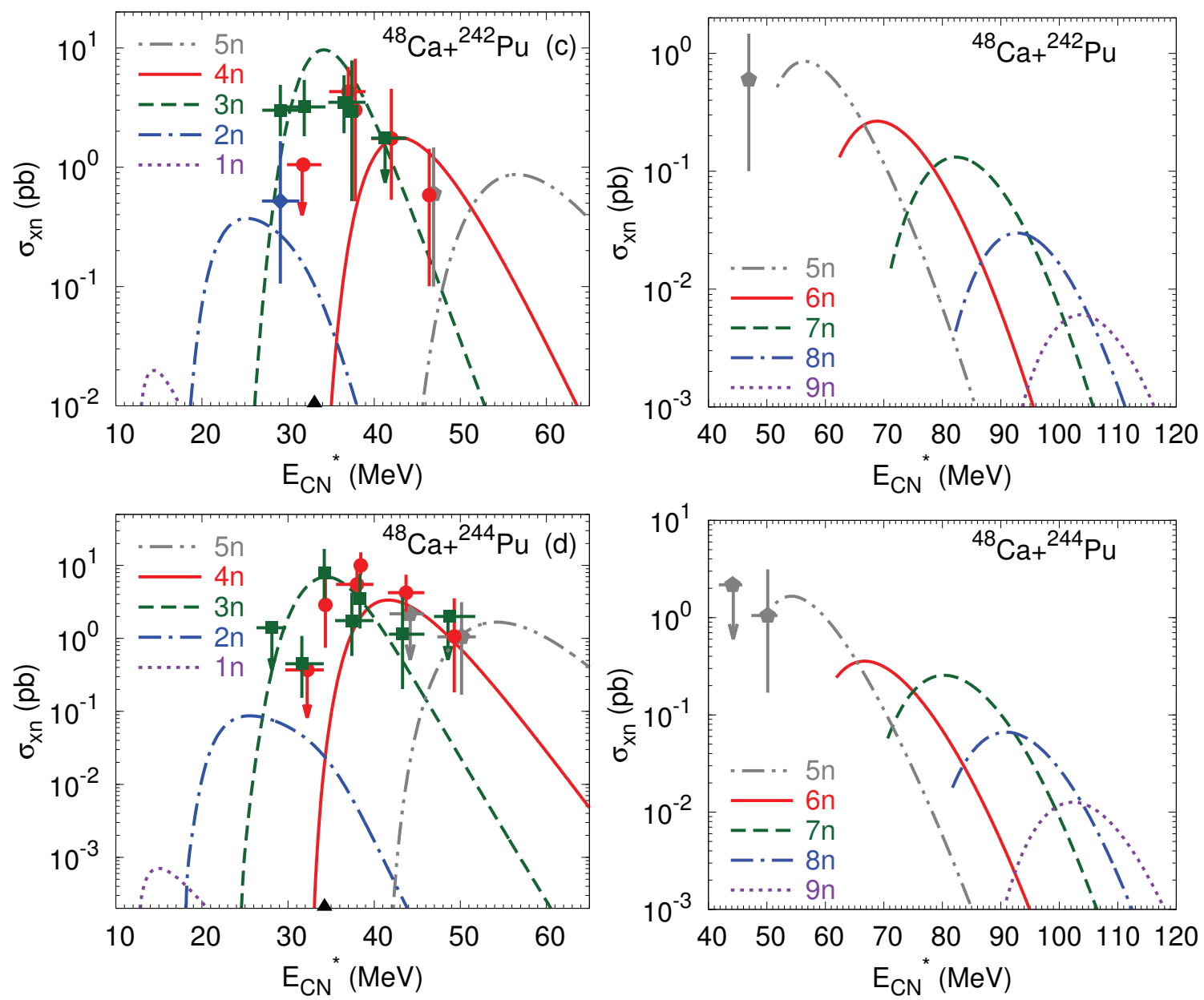

FIG. 3: The calculated (lines) excitation functions for $x n$-evaporation channels $(x=1-9)$ of the indicated complete fusion reactions. The mass table of Ref. [12] is used in the calculations. The mass numbers of isotopes produced are indicated. The black triangles at the energy axis indicate the excitation energy $E_{C N}^{*}$ of the $\mathrm{CN}$ at bombarding energy corresponding to the Coulomb barrier for the sphere-side orientation. The blue diamonds, green squares, red circles, and gray pentagons represent the experimental data [2] with error bars for $2 n-, 3 n-, 4 n-$, and $5 n$-evaporation channels, respectively. The symbols with the arrow indicate the upper limits of evaporation residue cross sections.

possibility, we have found, for the synthesis of new superheavy isotopes is related to alpha particles emission at the very beginning of the entire cascade process. Figure 6] shows the ratio of the widths $\Gamma_{n, p, \alpha}$ in the neutron, proton, and $\alpha$-particle emission channels to the total width $\Gamma_{t o t}$ as a function of the excitation energy for the different $\mathrm{Fl}$ isotopes. As seen, the emission widths of proton and $\alpha$-particle grow faster with increasing excitation energy 


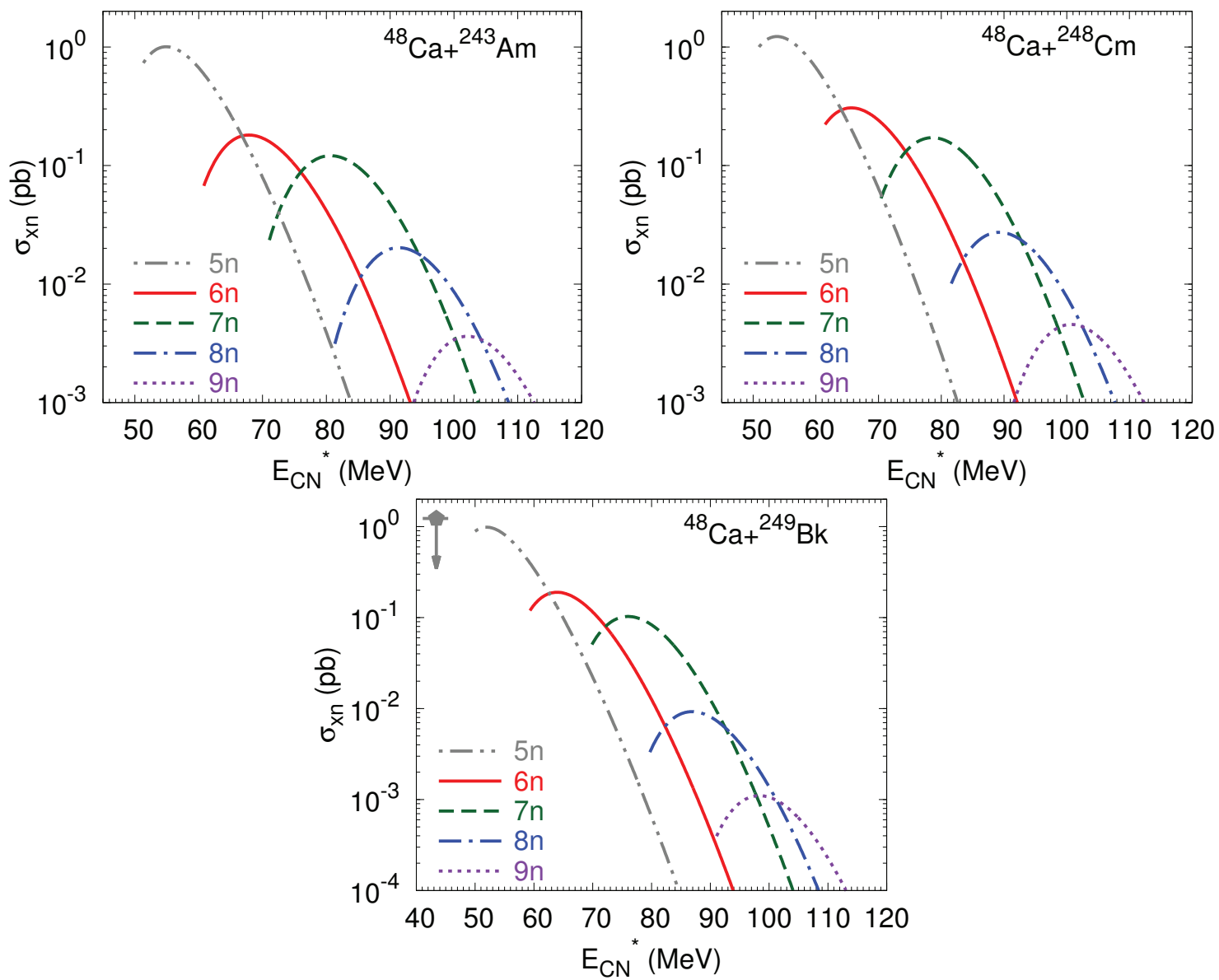

FIG. 4: The same as in Fig. 3, but for $x n$-evaporation channels $(x=5-9)$ of the indicated complete fusion reactions.

than the neutron emission width. At energies of the order and higher than $100 \mathrm{MeV}$, the emission widths of the neutron and $\alpha$-particle become of the same order, which leads to the suppression of the formation of nuclei in the neutron evaporation channel. The excitation functions of the $\alpha x^{\prime} n$-evaporation channels overlap with those from $x n$-evaporation channels.

For the ${ }^{48} \mathrm{Ca}+{ }^{244} \mathrm{Pu}$ reaction, we also present in Fig. 7 the total cross section

$$
\sigma_{t o t}\left(E_{\text {c.m. }}\right)=\sum_{x} \sigma_{x n}\left(E_{\text {c.m. }}\right)
$$

which is the sum of the cross sections of all neutron evaporation channels. With increasing excitation energy, from $E_{C N}^{*} \approx 12 \mathrm{MeV}$, the total cross section sharply increases by 5 orders of magnitude, reaches a maximum at $E_{C N}^{*} \approx 35 \mathrm{MeV}$, and then decreases relatively slowly at the $E_{C N}^{*}$ range of about $35-110 \mathrm{MeV}$. 

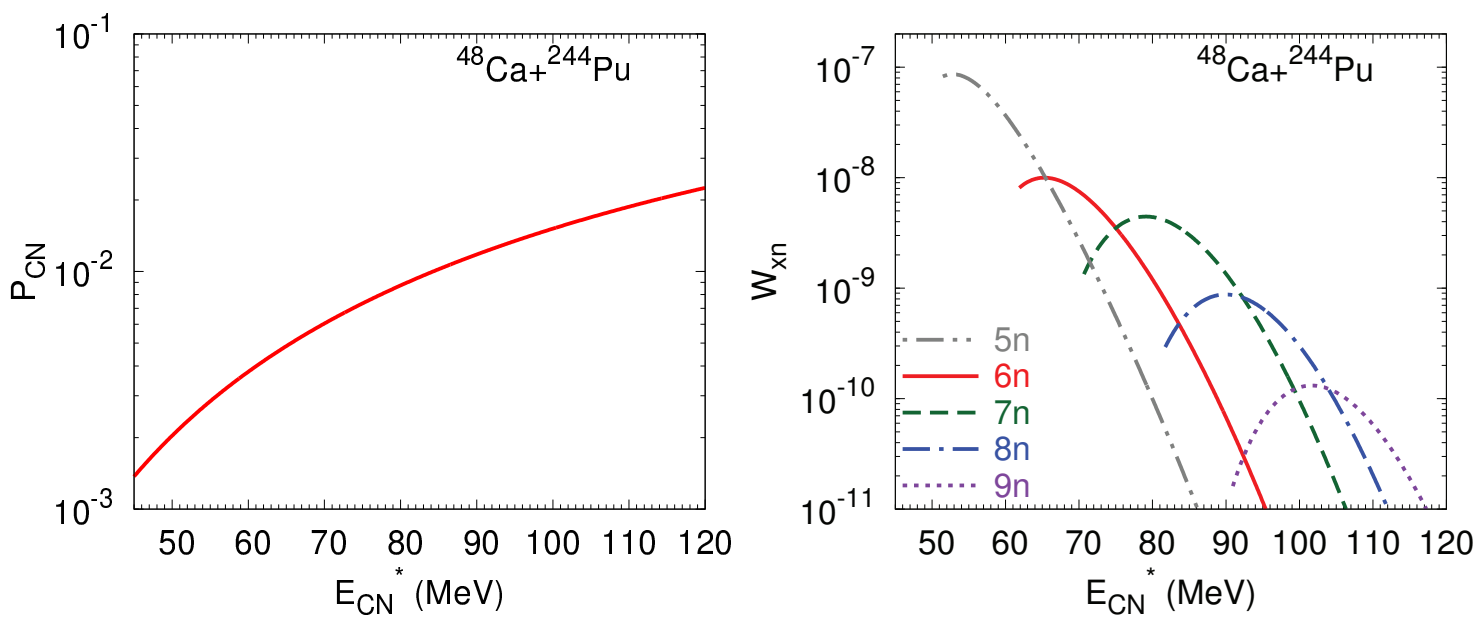

FIG. 5: The calculated fusion $P_{C N}$ and survival $W_{x n}(x=5-9)$ probabilities as a function of the excitation energy for the ${ }^{48} \mathrm{Ca}+{ }^{244} \mathrm{Pu}$ complete fusion reaction.

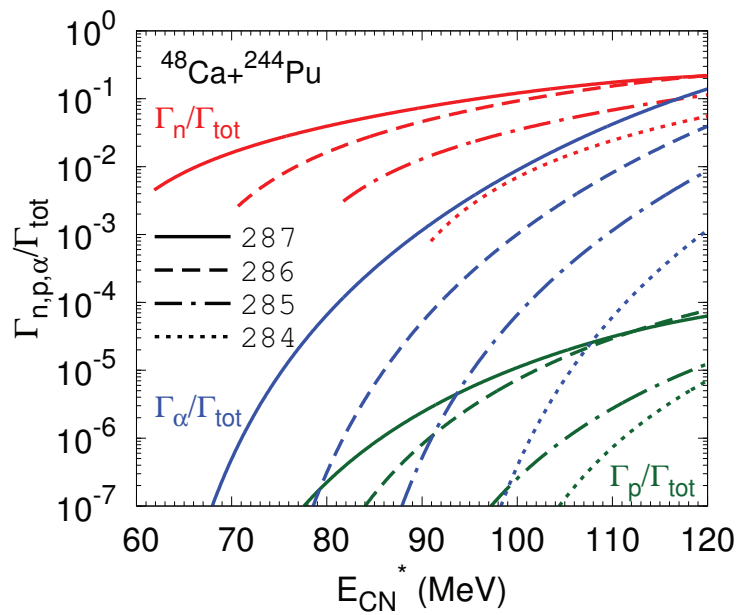

FIG. 6: The calculated ratios of the widths of neutron, proton, $\alpha$-particle emissions to the total width as a function of excitation energy for the nuclei ${ }^{284-287} \mathrm{Fl}$.

In conclusion, the excitation functions for the production of the new SHN with charge numbers $Z=114-117$ were calculated within dinuclear system model [11, 16], having strong predictive power, in $(5-9) n$-evaporation channels for the complete fusion reactions ${ }^{48} \mathrm{Ca}+{ }^{242,244} \mathrm{Pu},{ }^{243} \mathrm{Am},{ }^{248} \mathrm{Cm},{ }^{249} \mathrm{Bk}$. A very important element of these predictions is that they were made based on a uniform, consistent, and systematic set of input data. Predictions of global nuclear properties were done by using a well-tested multidimensional MM approach [12, 13]. In the presented letter, for the first time, we have indicated the possibility of producing new isotopes of the SHN in channels with high neutron 


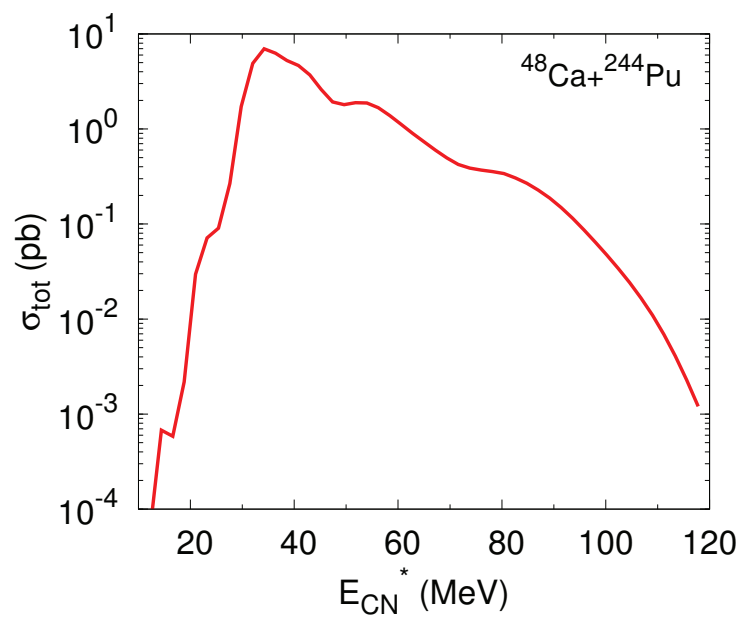

FIG. 7: The calculated total cross section as a function of the excitation energy for the indicated reaction.

multiplicities. As shown, the cross section drops down from about 1 pb to about $(1-10)$ $\mathrm{fb}$ at the transition from the $5 n$ - to the $9 n$-evaporation channel. Thus, the decline of the cross section with increasing excitation energy unexpectedly turned out to be relatively weak. This intriguing behavior may open up a new window for the study and the production of new isotopes of the SHN at high excitation energies. It would be interesting to compare the production cross sections in the hot and cold fusion reactions leading to the same neutron-deficient residual nuclei. With the planned increase of the beam intensity (of $\sim 10 \mathrm{p} \mu \mathrm{A}$ on target) [17] combined with the new Dubna gas-filled magnetic recoil separator (DGFS) setup [2], such extremely low cross sections will soon be measurable.

\section{Acknowledgments}

We are thankful to Prof. Yu.Ts. Oganessian for the initialization of this work and fruitful discussions. The work of G.G.A. and N.V.A. was supported by the Ministry of Science and Higher Education of the Russian Federation (contract 075-10-2020-117). M.K. was cofinanced by the National Science Centre under Contract No. UMO-2013/08/M/ST2/00257 
(LEA-COPIGAL).

[1] Yu.Ts. Oganessian, J. Phys. G 34, R165 (2007).

[2] Yu.Ts. Oganessian and V.K. Utyonkov, Nucl. Phys. A 944, 62 (2015); Rep. Prog. Phys. 78, $036301(2015)$.

[3] S. Hofmann and G. Münzenberg, Rev. Mod. Phys. 72, 733 (2000); S. Hofmann, Radiochim. Acta 99, 405 (2011).

[4] K. Morita et al., J. Phys. Soc. Jpn. 73, 2593 (2004); 76, 043201 (2007).

[5] A. Aprahamian, K. Langanke, and M. Wiescher, Prog. Part. Nucl. Phys. 54, 535 (2005).

[6] Yu.Ts. Oganessian et al., Phys. Rev. Lett. 104, 142502 (2010); Phys. Rev. C 87, 014302 (2013); 87, 034605 (2013); 87, 054621 (2013); V.K. Utyonkov et al., Phys. Rev. C 92, 034609 (2015); 97, 034609 (2018).

[7] S. Hofmann et al., Eur. Phys. J. A 32, 251 (2007); 48, 62 (2012); 52, 180 (2016).

[8] R. Eichler et al., Nature 447, 72 (2007).

[9] L. Stavsetra, K.E. Gregorich, J. Dvorak, P.A. Ellison, I. Dragojevic, M.A. Garcia, H. Nitsche, Phys. Rev. Lett. 103, 132502 (2009).

[10] Ch. Düllmann et al., Phys. Rev. Lett. 104, 252701 (2010); J.M. Gates et al., Phys. Rev. C 83, 054618 (2011); J.M. Khuyagbaatar et al., Phys. Rev. Lett. 112, 172501 (2014).

[11] J. Hong, G.G. Adamian, N.V. Antonenko, M. Kowal, and P. Jachimowicz, Phys. Lett. B 809, $135760(2020)$.

[12] M. Kowal, P. Jachimowicz, and J. Skalski, arXiv: 1203.5013; P. Jachimowicz, M. Kowal, and J. Skalski, At. Data. Nucl. Data. Tables. 138, 101393 (2021); DOI: 10.1016/j.adt.2020.101393.

[13] P. Jachimowicz, M. Kowal, J. Skalski, Phys. Rev. C 89 , 024304 (2014); 95, 014303 (2017).

[14] V.V. Volkov, Izv. Akad. Nauk SSSR, Ser. Fiz. 50, 1879 (1986); N.V. Antonenko, E.A. Cherepanov, A.K. Nasirov, V.B. Permjakov, and V.V. Volkov, Phys. Lett. B 319, 425 (1993); Phys. Rev. C 51 (1995) 2635.

[15] G.G. Adamian, N.V. Antonenko, and W. Scheid, Clustering effects within the dinuclear model, Lecture Notes in Physics, edited by C. Beck, 848, 165 (2012).

[16] J. Hong, G.G. Adamian, and N.V. Antonenko, Phys. Rev. C 92, 014617 (2015); 94, 044606 (2016); Eur. Phys. J. A 52, 305 (2016); Phys. Lett. B 764, 42 (2017); 805, 135438 (2020). 
[17] G.G. Gulbekian et al., Phys. Part. Nucl. Lett. 16, 866 (2019). 